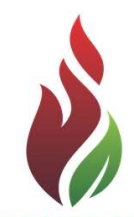

SUSTENERE

Publishing Corporation
ZRBADM

Journals Homepage:

www.sustenere.co/journals

\section{INVESTIMENTOS EM CAPACITAÇÃO EMPRESARIAL COMO BASE PARA IMPLEMENTAÇÃO DE INOVAÇÕES NAS EPP: UMA ANÁLISE DA CADEIA DE METAL MECÂNICA EM ARACAJU}

\section{RESUMO}

As pequenas empresas são vistas como mais abertas à inovação, no entanto ainda não se sabe quais áreas da pequena empresa podem favorecer a capacidade de inovação da empresa de forma mais relevante. Este estudo é de natureza quantitativa denominada survey, onde foram analisadas 31 empresas da cadeia de metal-mecânica da região da Grande Aracaju a luz do Radar da Inovação, buscando analisar a relação entre os investimentos em capacitação empresarial e a implementação de inovações na pequena empresa. a fim de responder a seguinte hipótese: Investimentos em cursos de capacitação auxiliam na implementação de inovação na pequena empresa? Os resultados encontrados mostram haver relação direta entre os fatores analisados. A conclusão do estudo foi afirmativa à hipotese da pesquisa, ressaltando a influência que os investimentos em capacitação possuem sobre a capacidade de implementação de inovações na pequena empresa, consequentemente no seu grau global de inovação e a influência que exerce no âmbito empresarial.

PALAVRAS-CHAVES: Inovação; Investimentos; Capacitação Empresarial; Pequenas Empresas.

\section{INVESTMENTS IN BUSINESS TRAINING AS THE BASIS FOR IMPLEMENTING INNOVATIONS IN EPP: AN ANALYSIS OF MECHANICAL METAL CHAIN IN SINGAPORE}

\section{ABSTRACT}

Small businesses are seen as more open to innovation, but it is not known which areas of small business can promote the innovation capacity of more relevant company. This study is called survey quantitative, which were analyzed 31 companies in the metal-mechanics of the Great regional chain Aracaju the light of the Innovation Radar, trying to analyze the relationship between investments in business training and implementation of innovations in small business . in order to answer the following hypothesis: Investments in training courses assist in the implementation of innovation in small business? The results show a positive relationship between the analyzed factors. The conclusion was affirmative to the hypothesis of the research, highlighting the influence that training investments have on the innovations of implementation capacity in small business, consequently in its overall degree of innovation and its influence in the business domain.

KEYWORDS: Innovation; Investments; Corporate Training; Small Business.
Revista Brasileira de

Administração Científica,

Aquidabã, v.6, n.1, Jan, Fev, Mar,

Abr, Mai, Jun 2014.

ISSN 2179-684X

SECTION: Articles

TOPIC: Empreendedorismo e Inovação

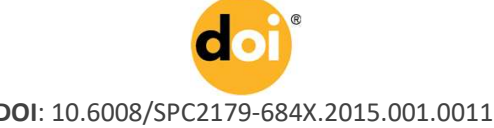

Carlos Cesar Santos

Universidade Federal de Sergipe, Brasil http://lattes.cnpq.br/8751536530803161 cordcesar@hotmail.com

Received: 26/09/2014

Approved: 14/10/2015

Reviewed anonymously in the process of blind peer.

Referencing this:

SANTOS, C. C.. Investimentos em capacitação empresarial como base para implementação de inovações nas EPP: uma análise da cadeia de meta mecânica em Aracaju. Revista Brasileira de

Administração Científica, Aquidabã, v.6, n.1, p.186 196, 2015. DOI: http://dx.doi.org/10.6008/SPC2179 684X.2015.001.0011 


\section{INTRODUÇÃO}

Diante dos diversos fenômenos ocorridos no ambiente organizacional capazes de conduzir a organização ao desenvolvimento, buscar compreendê-los é responsabilidade dos pesquisadores e dos dirigentes dedicados à gestão da inovação, afim de, melhorar o desempenho empresarial e promover a inovação organizacional dentro das empresas. Cruz (2003) esclarece que as pequenas empresas se caracterizam por serem mais inovadoras do que as grandes empresas, principalmente pela flexibilidade de adaptar-se rapidamente à demanda. No entanto a ausência de técnicas e conhecimentos formais das necessidades diárias da empresa dificulta a implementação das inovações diante da dinâmica organizacional particular apresentada pelas empresas de pequeno porte (EPP). Logo este estudo tem como objetivo, em âmbito geral, analisar a relação entre os investimentos em capacitação empresarial e a implementação de inovações nas EPP da cadeia de metal-mecânica da cidade de Aracaju. Este objetivo visa responder a seguinte hipótese: Investimentos em cursos de capacitação auxiliam na implementação de inovação na pequena empresa?

Para alcançar o objetivo geral e responder a hipótese da pesquisa estabeleceu-se como objetivos específicos: Fazer um levantamento teórico a respeito da temática, selecionar as empresas as serem estudadas, avaliar o desempenho das empresas perante o Radar Inovação em seus dois ciclos de aplicação RI0 (inicial) e o RI1 (Final), confrontar os resultados encontrados nas empresas estudadas, verificar se existe relação entre a hipótese levantada e os resultados encontrados no estudo. Embora o campo de estudo da gestão da inovação venha se desenvolvendo gradativamente nos últimos anos, ainda há necessidade de uma maior quantidade de alternativas e ferramentas que visem à compreensão e interpretação de situações peculiares à EPP, por suas especificidades não encontradas nas grandes empresas, nas quais a gestão da inovação torna-se mais visível, ganhando espaço principalmente nos departamentos de P\&D.

Sabendo que o Radar da Inovação (RI) foi desenvolvido, para ser capaz de analisar empresas de qualquer porte, a priori, aplicar este modelo a pequenas empresas, justifica-se pela oportunidade de consolidar, junto ao Serviço de Apoio a Micro e Pequenas Empresas (SEBRAE) como ferramenta de mensuração da inovação, e de desenvolver a capacidade da ferramenta enquanto método de análise científica da inovação nas EPP. Com o intuito de garantir a confiabilidade e o reconhecimento científico da pesquisa, esta se caracteriza como um estudo denominado survey, baseados nos dados colhidos pelo RI em empresas de pequeno porte da cadeia de metal mecânica da cidade em Aracaju. Dados estes, colhidos em campo pelos agentes locais de inovação (ALI).

O artigo está organizado em seções incluindo essa introdução, na segunda e terceira seções o texto trata de fundamentos teóricos sobre inovação nas pequenas empresas, capacitação empresarial e inovação. Na quarta seção são abordados os aspectos principais do programa ALI e do Radar da Inovação, na quinta seção trata-se sobre os aspectos metodológicos 
da pesquisa, a sexta seção é dedicada à análise dos dados obtidos. Na sétima seção trata da conclusão da pesquisa, seguindo-se das referências.

\section{REVISÃO TEORICA}

\section{A Inovação nas Pequenas Empresas}

A inovação tecnológica é um conceito muito difundido tanto no meio acadêmico como no meio empresarial e existe um consenso de que a inovação é o elemento de sucesso para a sobrevivência e competição das empresas, entretanto não existe um consenso referente à definição de inovação. A literatura apresenta diversos conceitos de inovação. A seguir, são apresentados alguns dos conceitos teóricos. Drucker (1986) define inovação como o ato de atribuir novas capacidades aos recursos (processos e pessoas) existentes na empresa para gerar riqueza. Dosi (1988) caracteriza a inovação como busca, descoberta, experimentação, desenvolvimento, imitação e adoção de novos produtos, processos e novas técnicas organizacionais.

Para Schumpeter (1934), a inovação pode ser a introdução de um novo produto ou mudança qualitativa em produto existente, a inovação de processo que seja novidade para uma indústria, abertura de um novo mercado, o desenvolvimento de novas fontes de suprimento de matéria-prima ou outros insumos e mudanças na organização industrial. Segundo Luecke (2003), a inovação é vista como sendo a incorporação e combinação de conhecimentos em algo original, relevante, como um novo produto, processo ou serviço. Para Girardi (2001), a inovação é resultado da criatividade utilizada pelas empresas na gestão de todos os seus processos.

O Manual de Oslo define a inovação como a implementação de um produto (bem ou serviço) novo ou significativamente melhorado, ou um processo, ou um novo método de marketing ou um novo método organizacional nas práticas de negócios, na organização do local de trabalho ou nas relações externas. O Manual de Oslo é a principal fonte internacional de diretrizes para coleta e uso de dados sobre atividades inovadoras da indústria (OCDE, 2005).

Brito et al. (2009) e Ferreira et al. (2007), afirmam que a inovação ocupa um papel de destaque na busca da competitividade das empresas. Porter (1996) afirma que uma empresa só poderá obter melhores resultados do que os seus concorrentes se conseguir criar um fator diferenciador que se mantenha ao longo do tempo, sendo o principal instrumento de criação dessa vantagem competitiva a inovação ou os atos de inovação. Segundo Tigre $(2006$, p.28), as empresas mais dinâmicas e rentáveis do mundo são aquelas mais inovadoras que, em vez de competir em mercados saturados pela concorrência, criam os seus próprios nichos e usufruem de monopólios temporários por meio de patentes e segredo industrial.

A inovação nas empresas depende diretamente dos processos inovativos por ela desempenhados, os quais proporcionam a competitividade das empresas. Segundo Lemes 
(1999), existe uma corrente da economia que corrobora com o pensamento de que os avanços resultantes de processos inovativos nas empresas são fator básico na formação da economia, bem como de seu desenvolvimento em longo prazo. Em acordo a esta linha de pensamento a Organização para a Cooperação e o Desenvolvimento Econômico (OCDE) afirma que a inovação é um dos fatores decisivos para o desenvolvimento econômico e social. Segundo a OCDE (2005), os indicadores de crescimento dos países mostram que a inovação contribui para o aumento da produtividade industrial e da qualidade de vida da população.

As pesquisas que tratam da questão da inovação nas empresas fazem uma ligação com as atividades de P\&D nas empresas, pois até pouco tempo a inovação era tida como um processo de modelo linear (LEMES, 1999), centrava-se na questão do P\&D como única fonte de inovação e não levavam em consideração outras atividades desenvolvidas nas empresas. Atualmente, outros aspectos foram incorporados como fontes de geração de inovação e reconhecidos como de papel relevante para o sucesso no processo de inovação nas empresas.

Baseado nesta afirmação, Santa María et al. (2008), desenvolveram um estudo, afirmando que o design, treinamento e o uso de máquinas e ferramentas avançadas são fontes de inovação em uma empresa. O Manual de Oslo (OCDE, 2005) lista algumas atividades além de P\&D como fontes de inovação nas empresas: aquisição de máquinas e equipamentos, atualização do ferramental, treinamento da equipe e até o marketing. $O$ treinamento é considerado uma atividade de inovação quando for necessário para a implantação de um produto ou processo tecnologicamente novo ou aprimorado. É fato a controvérsia existente na questão que trata a relação entre o tamanho da empresa e o desempenho inovador. Na visão de Hobday (1994 citado por LASTRES et al. 1998), de um modo geral as pequenas empresas não dispõem de ativos complementares necessários para explorar inovações em mercado de produção em massa e têm maiores dificuldades de obter resultados de suas inovações. As grandes empresas raramente dividem suas core capabilities dentro da rede, por questões econômicas, tecnológicas e estratégicas.

Cruz (2003) esclarece que as pequenas empresas se caracterizam por serem mais inovadoras do que as grandes empresas, principalmente pela flexibilidade de adaptar-se rapidamente à demanda. Em contra ponto, Barañano (2005), defende que não é o porte da empresa que a torna inovadora e sim outras características, tais como a estrutura da força de trabalho, a estratégia, as alianças com outras empresas ou com universidades e a organização interna da empresa. Silva et al. (2005) concluem em seu estudo de levantamento bibliográfico que a dimensão da empresa não é o fator determinante para a capacidade de invenção e inovação. Todavia, afirmam que geralmente as pequenas empresas obtêm melhor êxito nas inovações de produtos ou processo que as grandes empresas, apesar dos maiores investimentos em P\&D realizados pelas grandes. 


\section{Capacitação Empresarial e Inovação}

O conhecimento é um recurso crucial à competitividade econômica de empresas e nações no mercado mundial, seu domínio implica um trunfo para a conquista e a manutenção de nichos mercadológicos em condições de competição agressivas e imprevisíveis. Por isso, torna-se relevante a distinção entre os tipos de conhecimento e as habilidades estritamente necessárias para operar determinado sistema de producão de bens e serviços e aquelas requeridas para mudá-lo (Bell \& PAVITT, 1992). Kranzberg na década de 1980 já afirmava que, investimentos explícitos em aquisição e acumulação de conhecimento constituem o alicerce necessário à edificação da capacidade empresarial orientada para a geração de inovações em ambiente de crescentes exigências de competitividade de produtos e serviços. Países e empresas dispostos a desenvolver capacidade tecnológica com o propósito de acompanhar as tendências de transformações econômicas, sócio-culturais, políticas e tecnológicas têm pela frente o desafio de fomentar a contínua aprendizagem tecnológica (KRANZBERG, 1981).

A capacitação empresarial apresenta-se neste contexto com a ferramenta que auxilia a criação de capacidade para absorção de tecnologia, a aprendizagem é um processo que se desencadeia tanto no sistema de ensino formal e em instituições de pesquisa, como no âmbito das empresas. Uma de suas características é a estreita vinculação entre o setor tecno-científico, mais precisamente o universitário, e as empresas (CORREA, 1989).

A capacitação empresarial voltada à aprendizagem com foco na inovação trata-se de um desafio para empresas e países que pretendem tornar seus funcionários cidadãos aptos a se beneficiar dos avanços científico-tecnológicos e, mais do que isso, torná-los capazes de atuar como protagonistas dos processos de inovação tecnológica, a fim de melhorar os resultados das organizações. Nos países latino-americanos, o processo de aprendizagem se caracteriza preponderantemente pelo modelo de adaptação do indivíduo ao seu ambiente. Esse modelo, que se baseiam em critéríos, métodos e regras fixas para reagir frente a situações conhecidas e recorrentes, tem sido denominado 'aprendizagem de manutenção'(CORREA, 1989).

Obviamente, tal modelo de aprendizagem não pode ser totalmente descartado, visto que é e continuará sendo indispensável ao funcionamento e estabilidade de qualquer sociedade. Mas em épocas de rápidas mudanças e descontinuidade, como a de hoje, é imperativo introduzir um tipo de aprendizagem direcionada para capacitar indivíduos a lidar com situações imprevisíveis, em outras palavras, uma "aprendizagem inovadora" (Correa, 1989, p.119).

Para Correia, as organizações podem promover a aprendizagem inovadora, a partir de quando reconhecem a educação permanente como condição imprescindível para o estreitamento da 'brecha' que separa os países em desenvolvimento, e suas empresas, dos países desenvolvidos. Essa educação permanente implica: maior crédito à experiência e não somente à formação teórica; superação da clássica divisão da vida em etapas de aprendizagem e de ação, visto que ambas acontecem simultaneamente; e introdução de novas metodologias que capacitem 
indivíduos para aprender, reaprender continuadamente e, inclusive, para desaprender (CORREA, 1989).

Diante das contingências do mundo atual, a aceleração tecnológica pressupõe uma educação continuada. Esse tipo de educação, que ensina a aprender, enfatiza a capacitação para lidar com problemas em situações de incerteza (SIMONSEN, 1993). Defronte a isto a aprendizagem inovadora exige, portanto, uma nova concepção empresarial sobre a formação dos recursos humanos, baseada na interação empresa + educação + tecnologia (CORREA, 1989). É muito mais do que 'aprender-fazendo' ou "aprender-usando" ('learning by doing' ou 'learning by using'), que geralmente acontecem automaticamente. Implementar nas empresas a aprendizagem tecnológica com êxito, significa torná-la fruto de decisões conscientes e inovadoras empreendidas pela gerência, que, insatisfeita com o nível de eficiência alcançado em determinado momento, proporciona os recursos necessários para lograr inovações organizacionais (MEYER-STAMER, 1992).

Para um país se engajar com sucesso num processo de aprendizagem tecnológica, é imperativo o surgimento e a mobilização de um conjunto de empresas inovadoras (FAJNZLBER, 1983 citado por CORREA, 1989), capazes de implementar ações gerenciais estratégicas orientadas para a mudança tecnológica na empresa. Longe de substituir escolas e universidades, mas com relevante contribuição para o seguimento empresarial destaca-se o SEBRAE com soluções de capacitação e aprendizagem capazes de alavancar as micro e pequenas empresas, transformando-as em empresas inovadoras e melhorando seus resultados. Tais soluções apresentadas pelo SEBRAE são vistas por Correia como soluções que devem complementar a aprendizagem proporcionada pelo ensino formal e contribuir para a ampliação das capacitações dos indivíduos que nelas atuam (CORREA, 1989).

\section{O Programa Ali e o Radar da Inovação}

Com objetivo de aumentar a competitividade das pequenas empresas, por meio da difusão de informações sobre inovação, tecnologia e aplicação de soluções o programa Agente Locais de Inovação (ALI) foi criado pelo SEBRAE com a principal finalidade de fomentar a inovação nas MPE. Os Agentes Locais de Inovação são profissionais formados em diversas áreas de conhecimento e contratados como bolsistas pesquisadores em extensão no país pelo Conselho Nacional de Desenvolvimento Científico e Tecnológico (CNPq), os agentes têm o propósito de facilitar a busca de soluções para as demandas de cada empresa participante do programa, estimulando o desenvolvimento de processos, serviços e produtos inovadores.

O projeto é composto de seis etapas, que são: sensibilização de empresários para a adesão ao Projeto $\mathrm{ALI}$, adesão das empresas ao Projeto $\mathrm{ALI}$, aplicação do diagnóstico empresarial, aplicação do Radar da Inovação, elaboração do plano trabalho e implementação das ações demandas pelas empresas. O Radar da Inovação (RI) ferramenta adotada pelo SEBRAE para o acompanhamento da evolução da empresa durante o tempo de participação no programa 
ALI e ferramenta de mensuração adotada neste estudo, foi desenvolvido por Sawhney et al. (2006), com doze dimensões pelas quais uma empresa pode procurar caminhos para inovar. Posteriormente o RI foi complementado por Bachmann (2008) com uma décima terceira dimensão, ambiência inovadora, por entender que um clima organizacional propício à inovação é pré-requisito para inovar. Desta forma a ferramenta RI contempla as dimensões: Oferta; Plataforma; Marca; Clientes; Soluções; Relacionamento; Agregação de valor; Processos; Organização; Cadeia de fornecimento; Presença; Rede e Ambiência inovadora.

\section{METODOLOGIA}

O presente estudo é de natureza quantitativa denominada survey. A survey desenvolvida nesse artigo apresenta características de pesquisa descritiva, tendo seus dados coletados de maneira a defini-la como de caráter 'corte-tranversal' (FREITAS et al., 2000). A amostra estudada e a população alvo do estudo foram às empresas de pequeno porte da cadeia de metal-mecânica da grande Aracaju participantes do Programa ALI, por tanto, a amostra foi considerada não probabilística, sendo estas empresas atuantes apenas no setor do comercio a amostra foi considerada por conveniência.

O tamanho da amostra foi caracterizada por 31 empresas, tais empresas foram escolhidas por possuírem características semelhantes a fim de reduzir as incertezas na comparação dos resultados obtidos. Para isto as 31 empresas estudas fazem parte da cadeia de metal-mecânica da região da Grande Aracaju atuam no seguimento do comercio varejista e ingressaram no programa ALI no período de maio a julho de 2012. A ferramenta de mensuração utilizada como parâmetro de análise, com o objetivo de padronizar a informação e facilitar a posterior análise, foi o Radar da Inovação (RI) adotado como parâmetro no programa ALI. A estrutura do radar da inovação permite padronizar de forma numérica os resultados de cada organização, fator chave para a comparação dos resultados encontrados.

A aplicação da pesquisa se deu em duas etapas distintas, na primeira etapa, as empresas passaram pela primeira aplicação do Radar da Inovação (RI0) dentro de mesmo período, distanciando-se em 28 dias da aplicação da primeira para o da ultima empresa estudada, reduzindo assim possíveis interferências externas de cunho ambiental, político, internacional, etc. No segundo momento da pesquisa, seguindo o mesmo paramento as 31 empresas estudadas, passaram pela aplicação da segunda etapa do Radar da Inovação (RI1) dentro de um período de 30 dias, afim de manter a confiabilidade da comparação dos dados obtidos.

Em posse dos resultados das empresas a pesquisa seguiu duas linhas de comparação, na primeira, comparar a evolução da dimensão Ambiência Inovadora, que comporta os itens de fontes externas de conhecimento, entre o RI0 e o RI1. Na segunda linha de comparação, verificar se a evolução da dimensão Ambiência Inovadora reflete no grau global de inovação da empresa e por consequência na sua capacidade de implementar inovações. Por se tratar de um estudo onde apenas foi analisado um número muito reduzido de empresas, existem grandes limitações à 
generalização, por tanto, deve-se ressaltar que nem todas as EPP poderão identificar-se com os resultados apresentados neste artigo.

\section{Análise dos Dados}

Inicialmente as empresas estudadas foram reunidas em dois grupos, o Grupo 1, das empresas que realizaram investimentos em capacitação no período analisado e o Grupo 2, formado pelas empresas que não realizaram investimentos nesta área. Foram identificadas, dentre as empresas estudadas, como é apresentado no Gráfico 1, que 54\% das empresas estudadas realizaram investimentos em capacitação no período de avaliação do estudo. Percentual considerável, no entanto, demonstra que ainda é pouca a atenção destinada a capacitação pelos empresários do setor em analise.

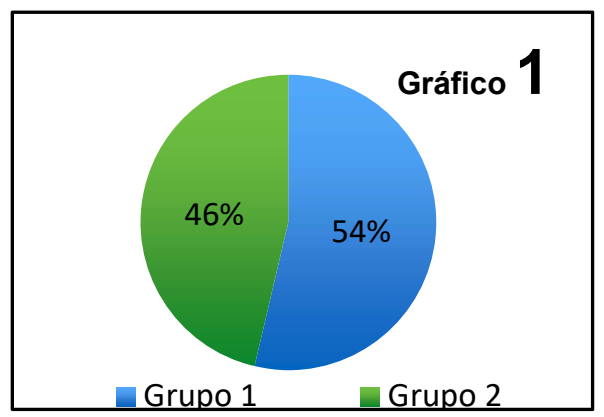

Gráfico 1: Empresas que realizaram investimento.

Como representado no Gráfico 2, que apresenta a media do grau global de inovação das empresas estudadas assim como a media do escore obtido na dimensão Ambiência Inovadora, pode-se evidenciar um paralelo entre o desempenho inovador das empresas e a promoção de uma ambiência inovadora de semelhante desempenho.

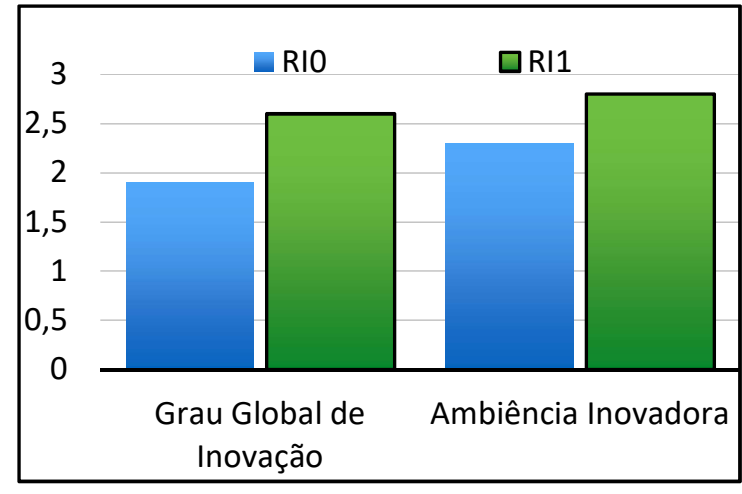

Gráfico 2: Media grau global.

Evidencia-se por meio da comparação dos resultados obtidos entre a primeira etapa de aplicação da pesquisa (RI0) e a segunda etapa de aplicação (RI1) que existe uma relação de crescimento entre os resultados, por comparação semelhante nos dois momentos em análise. 
Para confrontar o dados levantados na pesquisa os resultados das empresas foram agrupadas em quatro tipos: Tipo A: Média do escore alcançado pelas empresas, que não realizaram investimentos em capacitação, no Grau Global de Inovação (GGI) e na Ambiência Inovadora (Al), no primeiro momento da pesquisa (RI0); Tipo B: Média do escore alcançado pelas empresas, que realizaram investimentos em capacitação, no Grau Global de Inovação (GGI) e na Ambiência Inovadora (AI), no primeiro momento da pesquisa (RI0); Tipo C: Média do escore alcançado pelas empresas, que não realizaram investimentos em capacitação, no Grau Global de Inovação (GGI) e na Ambiência Inovadora (Al), no segundo momento da pesquisa (RI1); Tipo D: Média do escore alcançado pelas empresas, que não realizaram investimentos em capacitação, no Grau Global de Inovação (GGI) e na Ambiência Inovadora (AI), no segundo momento da pesquisa (RI1).

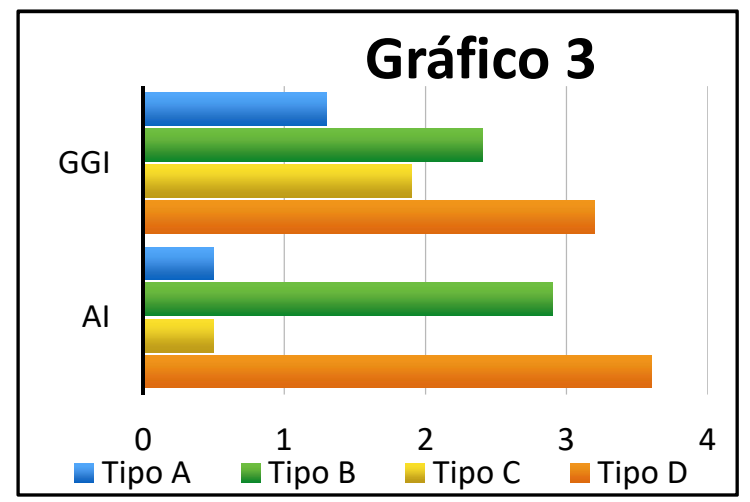

Gráfico 3: GGI.

Tendo sido confortados os resultados encontrados em cada agrupamento, pode-se observar, por meio do Gráfico 3, um aumento significativo do GGI médio das empresa que obtiveram aumento na dimensão ambiência inovadora, o que representa na prática empresarial que as empresas que investiram em conhecimento para alcançar melhorias e desenvolver inovações obtiveram um melhor êxito em suas demandas por desenvolvimento. As empresas que não investiram em capacitação e por consequência não obtiveram um bom desempenho na dimensão de ambiência inovadora, obteve um resultado pouco inovador em comparação as empresas que realizaram investimentos nessa área, o que evidencia a relação de forma inversa entre os investimentos em capacitação (conhecimento externos a empresa) e o sucesso na implantação de inovações.

\section{CONCLUSÕES}

Os resultados encontrados com este estudo demonstraram que empresas que realizaram investimentos em capacitação obtiveram maiores êxitos no desenvolvimento e na implantação de ações inovadoras, atingindo assim objetivo desde estudo e respondendo positivamente a hipótese apresentada. O grau de inovação global das empresas avaliadas demonstrou que as empresas que investem em capacitação estão buscando ações inovadoras para manter seu desempenho e 
suas posições no mercado de forma satisfatória, identificando, o que para o setor analisado, pode representar uma tendência, visto que, uma parcela de $54 \%$ das empresas analisadas foram caracterizadas como parcialmente inovadoras.

Consolida-se a relevância da pesquisa para o setor estudado e o Radar da Inovação com ferramenta de mensuração da inovação nas empresas, assim como a relevância do programa ALI, do Sebrae e da parceria com CNPq para o desenvolvimento da inovação no país. Destaca-se os $54 \%$ das empresas estudadas, que realizaram investimentos em capacitação como uma parcela representativa da amostra e sugere-se como pesquisa futura a análise das causas de $46 \%$ das empresas do seguimento não buscarem investir em capacitação e por consequência na implementação de inovações.

\section{REFERÊNCIAS}

BARAÑANO, A. M.. Gestão da inovação tecnológica: estudo de cinco PMEs portuguesas. Revista Brasileira de Inovação. Janeiro/Junho, 2005.

BELL, M.; PAVITT, K.. Accumulating technological capability in developing countries. Washington: Economics, 1992.

BESANKO, D. et al. Economics of Strategy. New York: Wiley, 2000.

BRITO, E. P. Z.; BRITO, A. L.; MORGANTI, F.. Inovação e seu Efeito no Desempenho das Empresas: Lucro ou Crescimento? RAE electron, São Paulo, v.8, n.1, 2009. DOI: http://dx.doi.org/10.1590/S1676$\underline{56482009000100007 .}$.

CORREA, C. M.. Tecnología y desarrollo de la informática en el contexto Norte-Sur. Buenos Aires: Eudeba, 1989.

CRUZ, R.. O empreendedor no processo de inovação de pequenas empresas de software do Rio Grande do Sul. In: EGEPE-ENCONTRO DE ESTUDOS SOBRE EMPREENDEDORISMO E GESTÃO DE PEQUENAS EMPRESAS. Anais. Brasília: UEM/UEL/UnB, 2003.

DOSI, G.. Sources, procedures and microeconomic effects of innovation. In: FREEMAN, C. (ed.).

Economics of Innovation. Aldershot: Edward Elgar Publishing, 1990.

DOSI, G.. The nature of innovative process. Technical change and theory. Londres: Pinter Publishers. 1988.

DRUCKER, P. F.. Inovação e espírito empreendedor. São Paulo: Pioneira, 1986.

FERREIRA, J. J. M.; MARQUES, C. S. E.; BARBOSA, M. J.. Relação entre Inovação, Capacidade Inovadora e Desempenho: O Caso das Empresas da Região da Beira Interior. Revista de Administração e Inovação, São Paulo, v.4, n.3, 2007.

GIRARDI, L. T. A.. Inovação e criatividade nas pequenas e médias empresas. Dissertação (Mestrado em Administração) - Fundação Getulio Vargas, Rio de Janeiro, 2001.

KRANZBERG, M.; PURCELL, C. W. J.. La importancia de la tecnologia en las cuestiones humanas. Barcelenona: Gustavo Gili S.A., 1981.

LASTRES, H.; CASSIOLATO, J.; LEMOS, C.; MALDONADO, J.; VARGAS, M.. Globalização e Inovação Localizada. Rio de Janeiro: Nota Técnica, 1998.

LEMES, C.. Informação e Globalização na Era do Conhecimento. Rio de Janeiro: REDESIST, 1999. 
LUECKE, R.. Managing Creativity and Innovation. Boston: Harvard Business School Press, 2003.

MANSFIELD, E.. Entry, Gibrat's law, innovation, and the growth of firms. American Economic Review, v.52, n.5, p.1023-1051, 1962.

MEYER-STAMER, J.. Capacidad tecnológica en países en vias de desarrollo: nuevos exigencias, nuevos enfoques. Berlin: Instituto Aleman de Desarrollo, 1992.

MOTOHASHI, K.. Innovation strategy and business performance of Japanese manufacturing firms. Economics of Innovation and New Technology, v.7, n.1, p.27-52, 1998.

OCDE. Organização para Cooperação Econômica e Desenvolvimento. Manual de Oslo: Proposta de Diretrizes para Coleta e Interpretação de Dados sobre Inovação Tecnológica. Tradução Financiadora de Estudos e Projetos. Brasília: Finep, 2005.

PORTER, M. E.. What is strategy? Harvard Business Review, Boston, v.74, n.6, p.61-78, 1996.

PORTER, M. E.. Vantagem competitiva. Rio de Janeiro: Campus, 1989.

SANTAMARIA, L.; NIETO, M. J.; BARGE-GIL, A.. Beyond formal R\&D: Taking advantage of other sources of innovation in low-and médium-technology industries. Madri: Research Policy, 2008.

SCHUMPETER, J. A.. The Theory of Economic Development. Cambridge Massachusetts : Harvard University Press, 1934.

SILVA, A. P.; JÚNIOR, E. P. A.; REIS, D. R.; LEITE, M. L. G.; PILATTI, L. A.. Inovação nas pequenas, médias e grandes empresas: vantagens e desvantagens. In: ENCONTRO NACIONAL DE ENGENHARIA DE PRODUÇÃO. 23. Anais. Ouro Preto: ENEGEP, 2005.

TIDD, J.. Innovation management in context: Environment, Organization and Performance. International Journal of Management Reviews, v.3, n.3, p.169-183, 2001.

SIMONSEN, M. H.. O que a educação deve ser para o século XXI.. São Paulo: Abril, 1993.

TIGRE, P. B.. Gestão da Inovação: A Economia da Tecnologia no Brasil. Rio de Janeiro: Elsevier, 2006. 